\title{
Dynamic transitions and hysteresis
}

\author{
Bikas K. Chakrabartia ${ }^{a, *}$ and Muktish Acharyya ${ }^{b, c,+}$ \\ ${ }^{a}$ Saha Institute of Nuclear Physics, 1/AF Bidhannagar, Calcutta-700 064, India \\ ${ }^{b}$ Institute for Theoretical Physics, University of Cologne, D-50923 Cologne, Germany
}

(October 3, 2018)

\begin{abstract}
When an interacting many-body system, such as a magnet, is driven in time by an external perturbation, such as a magnetic field, the system cannot respond instantaneously due to relaxational delay. The response of such a system under a time-dependent field leads to many novel physical phenomena with intriguing physics and important technological applications. For oscillating fields, one obtains hysteresis that would not occur under quasistatic conditions in the presence of thermal fluctuations. Under some extreme conditions of the driving field, one can also obtain a non-zero average value of the variable undergoing such "dynamic hysteresis". This non-zero value indicates a breaking of symmetry of the hysteresis loop, around the origin. Such a transition to the "spontaneously broken symmetric phase" occurs dynamically when the driving frequency of the field increases beyond its threshold value which depends on the field amplitude and the temperature. Similar dynamic transitions also occur for pulsed and stochastically varying fields. We present an overview of the ongoing researches in this not-so-old field of dynamic hysteresis and transitions.
\end{abstract}

Typeset using REVTEX 


\section{Contents}

I. Introduction

II. Magnetic response under an oscillating field:

Model studies

III. Dynamic hysteresis

IV. Dynamic transitions

A. Due to oscillating fields

B. Due to pulsed and stochastic fields

(a) Response to a positive pulse field

(b) Response to a negative pulse field and the

magnetisation-reversal transition

(c) Dynamic transition due to random

(time varying) fields

V. Concluding remarks

\section{INTRODUCTION}

Consider a co-operatively interacting many-body system, such as a magnet, driven by an oscillating external perturbation, such as an oscillating magnetic field. The thermodynamic response of the system, e.g., the magnetisation, will then also oscillate with necessary modifications in its form, and will lag behind the applied field due to the relaxational delay. This delay in the dynamic response gives rise to a nonvanishing area of the magnetisation-field loop, which phenomenon we term dynamic hysteresis. When the time period of oscillation of the external perturbation becomes much less compared to the typical relaxation time of the thermodynamic system, the hysteresis loop becomes asymmetric around the origin and an interesting thermodynamic phase arises spontaneously out of dynamically broken symmetries due to the competing time scales in such nonequilibrium driven systems. 
In the example of a magnetic system, the time $(t)$ variation of the magnetisation $m(t)$ lags behind that of the oscillating field $h(t)\left(=h_{0} \sin \omega t\right.$, say), and after some initial transient period, the dynamic $m(t)-h(t)$ loop stabilises and encloses a nonvanishing loop area $A\left(T, h_{0}, \omega\right)[=\oint m d h]$, which depends on the temperature $T$ of the system and the field amplitude $h_{0}$ and frequency $\omega$. This hysteresis is dynamical in origin and disappears in the quasistatic limit. Pure magnetic systems, without any random defects or anisotropies to pin the magnetic domains, can relax properly in the quasistatic limit and follow the field in phase due to the presence of thermal fluctuations at any finite temperature. No hysterisis can therefore occur in pure magnets in the quasistatic limit (Landau and Lifshitz 1935, Feynman et al 1964, Kittel 1966). It may be mentioned here that a subset of the engineering studies on hysteresis are of course in this quasistatic limit, in materials containing random pinning defects (see e.g., the Proc. Workshop on Hysteresis Modelling and Micromagnetism, Physica B 233 (1997) 259). Such quasistatic hysteresis in random materials has been modelled recently by using magnetic models having randomly quenched magnetic fields (Mirollo and Strogatz 1990, Sethna et al 1993). One studies here the self-organising avalanches in the zero-temperature spin-flip dynamics under external field (see e.g., Sethna et al 1993, Dahmen and Sethna 1993, Dhar et al 1997). For most of the design engineering problems connected with the recording processes and materials, the study of hysteresis is essentially dynamic in nature (see Torre 1966, Mallinson, 1987). A power law dependence of such hysteretic loss on the magnetic induction (related to the external field) was first proposed empirically, more than a century ago, by Steinmetz (1892). Here, in this colloquium, we confine ourselves to pure magnetic systems and examine the dynamic hysteresis arising out of two competing time scales, namely the relaxation time of the system and the time period of the external field. As such, it exists in any pure extensive (cooperative) system at any finite temperature, and disappears in the static limit. Following an early study of the dynamics in a driven model bistable system having rate competition (Agarwal and Shenoy 1981), the low frequency and amplitude power law behaviour for such dynamic hysteretic loss was first investigated in a multicomponent magnetic model by Rao et al (1989, 1990a), 
in the limit of a large number of components.

An important aspect of the magnetisation-field loop or $m-h$ loop shape, in this example of the dynamics of a pure magnet, is that the loop becomes asymmetric (in the positive and negative ranges of $m$ ) as the driving frequency of the field increases. The reason for this is that the system does not get enough time to relax, even to follow the sign (phase) of the external field. This causes a spontaneous symmetry breaking and a nonvanishing value of the dynamic order parameter $Q$ given by the time averaged magnetisation over a complete period $\left(Q=\frac{\omega}{2 \pi} \oint m d t\right)$. This non-zero value of the order parameter $Q$ develops spontaneously in the high frequency ranges, although the external perturbation does not provide, on average (over time), any symmetry breaking field $(\oint h d t=0)$. A prototype of this dynamic transition was first observed by Tomé and de Oliveira (1990) in the numerical solution of the mean field equation of motion of the classical one component magnetic model (see later in the next section). However, the transition there is not truely dynamic as it can exist for such equations of motion even in the zero frequency (static) limit of the driving field. This transition in the static limit is an artifact of the mean field approximation, which neglects nontrivial fluctuations. The occurrence of the dynamic transition for models incorporating thermodynamic fluctuations, was later shown in several Monte Carlo simulation studies (Lo and Pelcovits 1990, Acharyya and Chakrabarti 1994, 1995). In these numerical studies, incorporating fluctuations, the dynamic transition disappears in the static limit.

Although the subject of nonequilibrium phenomena associated with first order phase transitions has received considerable attention in the recent past, most of the attention has been focussed on two variants of the problem: Rapid quenching of a melt or of a spin system from a disordered phase to one of the competing ordered phases (see e.g., Bray 1994), or to the century-old problem of the decay (often remarkably slow, as for example in some allotropic forms of carbon) of the metastable phases of a condensed matter system through the nucleation of the domains of various phases, as the external conditions of the system are suddenly changed (see e.g., Rikvold and Gorman 1994). The interest in the study of nonequilibrium phenomena associated with extended systems, driven periodically or stochas- 
tically between two (or more) equivalent ordered phases, is somewhat more recent. These phenomena are also related to the phenomena of stochastic resonance in bistable systems which are periodically driven in the presence of stochastic noise (see e.g., Gammaitoni et al 1998). Here, the resonance of the weak periodic modulation (which by itself is incapable of inducing any phase change) with the Kramers' frequency of the theromodynamic system (coming from the finite temperature Boltzmann probability of activated hopping across a finite barrier between the two ordered phases) finally succeeds in inducing the periodic phase changes of the entire macroscopic system. Since its early introduction as a possible model for the dependence of the earth's ice age periods on the weak periodic modulations of the earth's dynamics through resonance of the stochastic noise of its weather (Benzi et al 1981), the concept has found wide applications to a variety of physical phenomena in biological and engineering sciences, including to dynamic hysteresis (Sides et al 1998a).

Interestingly enough, in spite of its immense practical importance in the engineering problems of magnetic recording, and the prospect of very interesting physics, the problem of dynamic transition and hysteresis in periodically driven magnetic systems was overlooked for many years. Although it is now less than ten years' old, the subject has become a field of considerable novelty and vitality. A number of key issues are already settled and a few others are nearly resolved. In this Colloquium we try to give an introduction to these intriguing nonequilibrium dynamic transition phenomena in driven extended systems and to discuss simple analytic theories connecting them.

\section{MAGNETIC RESPONSE UNDER AN OSCILLATING FIELD: MODEL STUDIES}

The detailed nature of the dynamic response of extended systems (having many interacting degrees of freedom), under time-dependent fields, is being investigated intensively these days. Considerable efforts have been made, in particular using computer simulations, to investigate the nature of the above mentioned dynamic phase transition and hysteresis in Ising models. 
Simple Ising systems contain ferromagnetically interacting spin degrees of freedom, each with binary (up/down or \pm 1 ) spin states. Let us consider a simple ferromagnetic system represented here by an Ising model with nearest neighbour ferromagnetic coupling, which is put under an oscillating external field. Such a system can be represented by the Hamiltonian

$$
H=-\sum_{<i j>} J_{i j} s_{i} s_{j}-h(t) \sum_{i} s_{i}
$$

with

$$
h(t)=h_{0} \sin \omega t .
$$

Here $s_{i}(= \pm 1)$ represents the Ising spin variable at the site $i$ on a $d$-dimensional lattice and $J_{i j}$ represents the spin-spin interaction strength between sites $i$ and $j . \sum_{i}$ runs over all the lattice sites and $\sum_{<i j>}$ runs over all the distinct nearest-neighbour pairs. Note that for truly long-range interactions, as in the mean field models discussed later, the sum in (1) extends over all the pairs and the value of $J_{i j}$ decreases inversely with the number of sites in the system. The system is in contact with an isothermal heat bath at temperature $T$. For simplicity all $J_{i j}(>0)$ are taken equal to a constant $J(=1)$, and the temperature $T$ is measured in units of $J$, setting the Boltzmann constant at unity. In order to keep $H / T$ dimensionless, $h_{0}$ in (1a) is also measured in units of $J$.

We will discuss later the equations of motion for the average magnetisation $m\left(=\left\langle s_{i}\right\rangle\right.$, where $\langle\ldots\rangle$ denotes the ensemble average) for such systems. It may be noted here that since no transverse magnetisation is possible for the Ising system (the magnetisation $m$ is a scalar), the magnetisation can only try to follow the field, albeit with a delay. The behaviours of the response magnetisation $m(t)$ are qualitatively indicated in Fig. 1 for some typical cases. Generally, since the equation of motion of such systems remains invariant for $t \rightarrow t+2 \pi / \omega$, the response magnetisation also becomes periodic (not necessarily sinusoidal, even though the field is so) with the same periodicity or some integer multiple of it (in cases of stochastic resonance). This is responsible for the feature that the magnetisation 
$m$ can at most be double-valued at any field $h$, and the $m-h$ loop has to be closed (on average; in the thermodynamic limit). The phase delay in $m(t)$ (compared to that of $h(t)$ ) gives rise to the dynamic hysteresis loss or the loop area $A$. The same delay and the constraint of identical periodicity for the field and the response magnetisation can induce an asymmetry in the response (in $\pm m$ ) as the driving frequency increases. When the time period of oscillation falls far behind the effective relaxation time of the many-body system, a dynamically broken symmetric phase arises spontaneously with a nonvanishing value of the dynamic order parameter $Q$ (the period averaged magnetisation).

Typically, for fixed temperature $T$ and field amplitude $h_{0}$, the dynamic hysteresis loss $A$ increases with increasing frequency $\omega$ for low values of $\omega$. This is because, for low values of $\omega$, the effective delay in the response increases as $\omega$ increases. In general, for a fixed $\omega, A$ increases with decreasing $T$ and increasing $h_{0}$ until $A$ saturates. Eventually, as the driving frequency exceeds a threshold value (dependent on $h_{0}$ and $T$; see Acharyya 1998b), the loop area $A$ starts decreasing, because of the increase in the effective delay (phase lag) towards $2 \pi$. Eventually, the loop area vanishes for very high frequencies when the dynamic symmetry is fully broken $(Q=1)$. For a fixed frequency, this dynamic transition phase boundary (seperating non-zero and zero values of $Q$ in the $h_{0}-T$ plane) is in general convex towards the origin. With large values of the field amplitude $h_{0}$ or temperature $T$, one gets a "forced oscillation" kind of scenario inducing the dynamically symmetric $(Q=0)$ phase. However, right near the dynamic phase boundary, in the symmetric $(Q=0)$ phase, the field amplitude $h_{0}$ may not be sufficient to cross the free energy barrier at that temperature and stochastic resonances can induce such dynamic symmetry in several attempts or in averages over several cycles (Sides et al 1997, 1998a). With decreasing $\omega$, the phase boundary shrinks inward. Finally, in the static limit $(\omega=0)$ the dynamic transition disappears and the phase boundary in the $h_{0}-T$ plane collapses to a line with $h_{0}=0$ and ending at $T=T_{c}$, the static transition temperature of the unperturbed system.

The majority of studies on such Ising systems in an oscillating field have been made employing the Monte Carlo method (see e.g., Binder 1979) using the Metropolis single spin 
flip dynamics. Starting from an arbitrary initial state or configuration of spins (e.g., with all spins up), the spin state $s_{i}(t)$ at each site $i$ and time $t$ is updated sequentially with a probability proportional to $\exp \left[-\frac{1}{T} \Delta E_{i}(t)\right]$ where $\Delta E_{i}(t)=2 s_{i}\left[\sum_{j} s_{j}(t)+h(t)\right]$, the change in energy due to the spin flip. One full scan over the entire lattice is defined as the unit time step (or Monte Carlo step per site). The response magnetisation (per site) at time $t$ is then easily calculated: $m(t)=(1 / N) \sum_{i} s_{i}(t)$, where $N$ is the total number of spins in the lattice $\left(N=L^{d}\right.$ if one considers a $d$-dimensional hypercubic lattice of linear size $\left.L\right)$. One then concentrates on the behaviour of the response function $m(t)$ for a long time $t$ (much after the stabilisation, starting from the initial state) as compared to the field $h(t)$. Specifically, one considers quantities like the loop area $A(=\oint m d h)$ and the dynamic order parameter $Q\left(=\frac{\omega}{2 \pi} \oint m d t\right)$

The mean field equation of motion for the average magnetisation $m$ can be written as

\footnotetext{
${ }^{1}$ In a cooperatively interacting system as, for example, described by the Hamiltonian (1), each degree of freedom (here spin) interacts with the neighbouring one. Hence the interaction tries to induce complete order $(m=1)$ in the system. The thermal noise, at any nonvanishing $T$, induces fluctuations destroying the order and takes the system to microscopic states which are not energetically favourable. The probabilities of such higher energy states are given by the Boltzmann probabilities at that $T$. The overall order is then given by the thermodynamic average value $(m<1)$, while there are fluctuations around this average. In the mean field approximation, one isolates an arbitrarily chosen cluster of spins (usually one spin) and performs the statistical mechanics for these chosen spins exactly, taking the states of all its (interacting) neighbours at their mean or average state (represented by the average $m$ ). This self-consistent approximation therefore pictures each degree of freedom to be placed in a mean field provided by the average state of the neighbours and the interaction strength $J$ (in (2) the value of $T_{c}=J Z_{n n}$ has been put equal to unity, where $Z_{n n}$ denotes the number of nearest neighbours of any spin).
} 


$$
\frac{d m}{d t}=-m+\tanh \left(\frac{m+h(t)}{T}\right)
$$

where $h(t)$ is given by (1a) and the microscopic relaxation time (on the right hand side of (2)) is put equal to unity (see Suzuki and Kubo 1968). This simple nonlinear equation (in one variable) is indeed capable of capturing a number of important features of dynamic hysteresis and of the dynamic transition. However, the lack of fluctuations of the thermodynamic average value $m$ in the above equation is responsible for the loss of some very significant features. For example, even in the quasistatic limit, one requires a nonvanishing coercive field amplitude to overcome the free energy barrier or to go from a $+m$ state to the corresponding $-m$ state crossing the barrier which is finite below the static order-disorder transition temperature $T_{c}$. Consequently, a nonzero hysteresis loop area can be found even in the static limit. Also, any applied field here (of magnitude below that of the coercive field which is nonzero for $T<T_{c}$ ) cannot break the asymmetry of the system. The "dynamic transition" therefore occurs here even in the static limit! Still, considerable analytical studies have been made for hysteresis from such single-variable, nonlinear, periodically-driven equations of motion or maps (Jung et al 1990, Goldsztein et al 1997). Some efforts have been made to incorporate the effects of Gaussian noise on the Langevin type equation of motion for $m$ with a somewhat weaker nonlinearity (Mahato and Shenoy 1994, Paniconi and Oono 1997). Efforts have also been made to treat the effects of fluctuations using a renormalisation group technique for a general $n$-component system (with $n$ components for

the magnetisation vector $m$ ), and study the $n \rightarrow \infty$ limit (Rao et al 1990a,b, Rao and Pandit 1991, Thomas and Dhar 1993, Fan and Jinxiu 1995).

\section{DYNAMIC HYSTERESIS}

The dynamic contribution to the coercive field and the hysteresis loop area has been investigated in several recent experiments, mostly in thin films or in two dimensions. Bruno et al 
(1990) studied the dependence of the hysteresis loop area $A$ on the rate of change of the external field, in ultrathin ferromagnetic films. Their study gives some indirect information on the dynamic contribution to the loop area $A$. In a more recent experiment, Jiang et al (1995, 1996a,b) studied the frequency-dependent hysteresis of epitaxially grown ultrathin (2 to 6 monolayer thick) Co films on a $\mathrm{Cu}(001)$ surface at room temperature. The films have strong uniaxial magnetisation with two ferromagnetic phases of opposite spin orientations. This magnetic anisotropy makes it appropriate to represent the system by an Ising-like model (see also He and Wang 1993). The external magnetic field $h(t)$ on the system was driven sinusoidally in the frequency $(f=\omega / 2 \pi)$ range 0.1 to $500 \mathrm{~Hz}$ and in the amplitude $\left(h_{0}\right)$ range 1 to 180 Oe. Here of course the time-varying current or the magnetic field induces an eddy current in the core, which results in a counter-field reducing the effective magnitude of the applied field. The surface magneto-optical Kerr effect technique was used to measure the response magnetisation $m(t)$. A typical variation of the loop area $A$ with the driving frequency $f$, at room temperature and at fixed external field amplitude $h_{0}$, is shown in Fig. 2a. Also, it may be mentioned that in a recent similar experiment on dynamic hysteresis in ultrathin Fe films on W(110) surface (Suen and Erskine 1997), the typical behaviour of the dynamic hysteresis is observed to be similar, although considerable discrepancies are observed in the actual details for different materials and regimes.

The observed variation of the loop area $A$ with frequency $\omega$ follow the generic form discussed earlier: $A$ decreases for both low and high values of $\omega$. However, it may be noted that $A$ does not quite vanish in the zero frequency limit. The observed variation can, in fact, be fitted to a form

$$
A=A_{0}+h_{0}^{\alpha} \omega^{\beta} g\left(\frac{\omega}{h_{0}^{\gamma}}\right)
$$

with the scaling exponents $\alpha, \beta$ and $\gamma$ and with the scaling function $g$ having a suitable 
nonmonotonic form such that $g(x) \rightarrow 0$ as $x \rightarrow 0$ or $\infty$. Here $A_{0}$ is the loop area in the zero frequency limit. It seems, depending on the nature of the dynamic processes involved in different materials and also the ranges (of amplitude, frequency, etc), the values of the exponents differ dramatically. While Jiang et al (1995) obtained $\alpha \simeq 0.67 \simeq \beta$ for Co films on $\mathrm{Cu}(100)$, and $\alpha \simeq 0.6$ and $\beta \simeq 0.3$ for Fe films on $\mathrm{Au}(001)$, Suen and Erskine (1997) obtained much lower values for the same exponents: $\alpha \simeq 0.3$ and $\beta \simeq 0.06$ (perhaps logarithmic) for Fe films on W(110) surfaces. It may be mentioned that Jung et al (1990) observed, in the context of their analytical study for a model switching between two modes of a semiconductor laser, that one needs to replace $h_{0}$ in $(3)$ by $\tilde{h}_{0}$, to account for a subtraction of a threshold field amplitude below which no hysteresis takes place.

This experimental observation perhaps indicates that the observed hysteresis is not entirely dynamic in origin; $A$ does not quite vanish in the static limit $\omega \rightarrow 0$. An approximate mean field solution of (2) can of course give the above fitting form (3) for $A$ (Jung et al 1990, Luse and Zangwill 1994, Hohl et al 1995, Goldsztein et al 1997): Linearizing the mean field equation (2), for small $m$ and $h_{0}\left(T>T_{c}=1\right)$, one gets

$$
\frac{d m}{d t}=-\epsilon m+\frac{h(t)}{T} ; \quad \epsilon=\frac{T-1}{T} .
$$

The steady state solution of which can be written as $m(t)=m_{0} \cos (\omega t-\phi)$ for

2 The constants $\alpha, \beta, \gamma$, etc, in general assume noninteger values, indicating singularities in the power laws. Very small values of the constants are often taken as indications for logarithmic variations rather than power laws (taking the representation $\left.\ln x=\left[\left(x^{n}-1\right) / n\right]_{n \rightarrow 0}\right)$. These constants are called the scaling exponents here, in conformity with the practice in critical phenomena, where the exponents also turn out to be universal in the sense that they do not depend on most of the details of the thermodynamic system. The scaling funcition is again a term borrowed from the critical phenomena literature, where the function $g$ is not dependent on the individual values of $\omega$ and $h_{0}$, but is a function only of the scaled variable $\omega / h_{0}^{\gamma}$. 
$h(t)=h_{0} \cos \omega t$. A direct substitution then gives $m_{0}=h_{0} / T \sqrt{\epsilon^{2}+\omega^{2}}$ and $\phi=$ $\sin ^{-1}\left(\omega / \sqrt{\epsilon^{2}+\omega^{2}}\right)$. For the loop area $A$ in this linearised limit, one gets

$$
\begin{gathered}
A=\oint m d h \sim h_{0}^{2} g(\omega) / T ; \\
g(\omega)=\frac{\omega}{\epsilon^{2}+\omega^{2}} .
\end{gathered}
$$

The above Lorentzian form for the variation of the loop area $A$ with frequency $\omega$ is in fact valid for $T>T_{c}$. For $T \lesssim T_{c}$ a similar form for the frequency variation will be valid with an additional frequency-independent contribution $A_{0}(T) \sim m_{e}(T) h_{c}(T) \sim\left(T_{c}-T\right)^{2}$, where $m_{e}(T)$ denotes the equilibrium magnetisation and $h_{c}(T)$ the static coercive field at that temperature $T<T_{c}$ (=1 here). The above Lorentzian form (5) for the variation of the dynamic hysteresis loop area $A$ with the frequency $\omega$ of the driving field, at high temperatures, may be compared with that given in (3) with $\alpha=2, \beta=1$ and $\gamma=0$. In fact, the numerical solution for the loop area $A$ from (2) for $T>T_{c}(=1)$ gives excellent agreement with (5) ( $A_{0}$ is zero for $\left.T>T_{c}\right)$. At higher field amplitudes and lower temperatures $\left(T<T_{c}\right)$, the above linearisation of the tanh term in (2) becomes inappropriate. Jung et al (1990) and Goldsztein et al (1997) considered the effect of an additional $m^{3}$ term in the right-hand side of (4). In the limit $\omega \rightarrow 0$, they could solve such a nonlinear equation and obtained $A \sim A_{0}+\tilde{h}_{0}^{a} \omega^{b}$ with $a=2 / 3=b$ for positive values of $\tilde{h}_{0}=h_{0}-h_{c}$.

In models for order-disorder ferroelectrics, the co-operative dipole-dipole interaction is represented by an Ising Hamiltonian, while the quantum tunnelling between the two equivalent wells (corresponding to Ising states \pm 1 ) is represented by a (non-commuting) tunnelling field. A similar mean field treatment of the semi-classical equation of motion of a quantum (Ising) system, for finite temperature hysteresis, was performed by Acharyya et al (1994). Here the tunnelling field is driven sinusoidally to simulate a periodic modulation of the external pressure on the sample.

All these mean field studies, mentioned above, are for systems without any thermal fluctuations. In any realistic system, as in the experimental systems described above, such fluc- 
tuations are present and theoretical analysis becomes considerably more difficult. However, computer simulation studies on such models are possible and these studies give significant insights regarding the effects of such fluctuations. Extensive Monte Carlo studies have been made for the Ising system represented by the Hamiltonian (1) in one to four dimensions (Lo and Pelcovits 1990, Acharyya and Chakrabarti 1994, 1995). Acharyya and Chakrabarti observed that at high temperatures $\left(T>T_{c}\right)$ the loop area variation for the entire frequency range can be represented by

$$
\begin{gathered}
A\left(T, h_{0}, \omega\right) \sim h_{0}^{\alpha} T^{-\rho} g\left(\frac{\omega}{h_{0}^{\gamma} T^{\delta}}\right), \\
g(\tilde{\omega}) \sim \tilde{\omega}^{\beta} \exp \left(-\tilde{\omega}^{2} / \sigma\right),
\end{gathered}
$$

where the scaling function $g(\tilde{\omega})$ is exponentially decaying in such Monte Carlo studies, with an initial power-law growth (compared to the Lorentzian form (5) in the linearised mean field case). The above scaling form (6) obviously reduces to a power law in the low frequency limit: $A \sim h_{0}^{a} \omega^{b} T^{-c}$, with $a=\alpha-\beta \gamma, b=\beta$ and $c=\rho+\beta \delta$. The fitting curve for the collapsed data for the loop area $A$ as function of the scaled frequency $\tilde{\omega}\left(=\omega / h_{0}^{\gamma} T^{\delta}\right)$ gives the scaling function $g(\tilde{\omega})$. The best fit values of the exponents $\alpha, \beta, \gamma$ and $\delta$ depend on the dimension. One typical result for the data collapse for $A$ in two dimensional Monte Carlo study is shown in Fig. 3. The best fit values for these exponents seem to be $\alpha \simeq 1.0$; $\beta \simeq 0.3,0.5$ and $0.5 ; \gamma \simeq 0.9,0.7$ and $1.4 ; \delta \simeq 1.2,1.8$ and $1.4 ;$ and $\rho \simeq 0.8,1.2$ and 0.5 , in $d=2,3$ and 4 respectively. This gives $a \simeq 0.7,0.6$ and 0.3 , and $b \simeq 0.3,0.5$ and 0.5 respectively in the above dimensions (Acharyya and Chakrabarti 1995). For linearly swept fields, extensive simulation studies to check the above scaling behaviour of $A$ has been done recently by Zheng and Zhang (1998a,b). They estimated the value of the dynamic exponent $z$ (see section IV B), from the exponent $\beta$ and compared that with the direct Monte Carlo estimates for the same.

If the applied field amplitude and the system size are both small, one can utilise a picture 
for the spin flips occurring through the nucleation of a single spin domain (Thomas and Dhar 1993, Sides et al 1998a). The classical nucleation theory of Becker and Döring (see e.g., Rikvold and Gorman, 1994) suggests that the nucleation rate is $I \sim \exp \left[-F\left(l_{c}\right) / T\right]$, given by the optimality condition $l_{c}=[\sigma(d-1) / 2 d] / h$ of the free energy $F(l)=2 h l^{d}+\sigma l^{d-1}$ for the formation of a droplet or domain of linear size $l$ under field $h$. Here $\sigma$ is proportional to the surface tension for the formation of the droplet. For low temperatures the magnetisation switches from about +1 to -1 as the field is swept from positive to negative values. The hysteresis loop area $A$ is then essentially proportional to the (dynamic) coercive field $h_{c} \simeq$ $h_{0} \omega t_{s}$, where the switching time $t_{s}$ can be estimated by requiring an order of unity value for the total integrated switching probability within $t_{s}$ :

$$
\begin{gathered}
\int_{0}^{t_{s}} d t \exp \left(-\frac{1}{[h(t)]^{d-1}}\right) \\
\simeq\left(h_{0} \omega\right)^{-1} \int_{0}^{h_{c}} d h \exp \left(-\frac{1}{h^{d-1}}\right)=1 .
\end{gathered}
$$

This suggests that

$$
A \sim h_{c} \sim\left[-\ln \left(h_{0} \omega\right)\right]^{-1 /(d-1)}
$$

for small $t_{s}$ and $\omega$. In this estimate, the loop area disappears logarithmically in the static limit. Extensive simulation studies by Sides et al (1998a,b) and by Acharyya and Stauffer (1998) for small system sizes and low field amplitudes seem to suggest that the above logarithmic dependence on frequency can indeed be observed for extremely low frequencies. However the inaccessibility of such really low frequencies, both in simulations and in experiments, and power law type fits to the data for rather high frequencies might be responsible for the apparent indication of an effective constant value $A_{0}$ for the "extrapolated" loop area in the zero frequency limit, as discussed earlier in (3). Let us remember that such an analysis is valid only when a single domain of flipped spins grows and induces the switching in magnetisation. As the field amplitude increases, and/or the system size becomes much 
larger than the single critical domain size $l_{c}$, the switching occurs by the coalescence of multiple domains. The extension of the above nucleation rate analysis from a single domain to the crossover region of multi-domains has also been done recently (Sides et al 1998b,d; see also Sides 1998). In such strong field cases, however, the power law scaling behaviour (6) seems to be quite appropriate as indicated by the results from various Monte Carlo studies (Acharyya and Chakrabarti 1995). Analytically, the effect of such fluctuations can still be tackled in the large $n$ limit of an $n$-vector model (Rao et al 1990a), for which one finds $A \sim h_{0}^{a} \omega^{b}$ for $\omega \rightarrow 0$ in the high temperature limit, with $a=1 / 2=b$ with logarithmic corrections (Dhar and Thomas 1992, see also Fan and Jinxiu 1995).

\section{DYNAMIC TRANSITIONS}

\section{A. Due to oscillating fields}

As mentioned in the preceding section, the experiment by Jiang et al (1995) on dynamic hysteresis in epitaxially grown ultrathin Co films on a $\mathrm{Cu}(001)$ surface with magnetic anisotropy at room temperature exhibits a prominent signature of the dynamic transition as the driving frequency increases. The observed hysteresis loops tend to become asymmetric about the zero magnetisation line (see Fig. 2a) in the high frequency regime. Even for a very low frequency $(f=4 \mathrm{~Hz})$, they observed the same symmetry breaking dynamic transition by reducing the amplitude of the magnetic field (see Fig. 2b). However, the precise experimental phase boundary for this dynamic transition is not available to date. Moreover, no experimental attempt has been made so far to probe the thermodynamic nature of such dynamic transitions. Similar a dynamic transition can also be clearly seen from the data obtained by Suen and Erskine (1997) in Fe films on W(110) surfaces.

\section{Mean field scenario:}

Tomé and de Oliveira (1990) studied the response of a kinetic Ising model in the presence of a sinusoidally oscillating magnetic field, by solving the mean field equation of motion (2) for 
the average magnetisation. The dynamic order parameter $Q$ (the time average magnetisation over a full cycle of the oscillating field), which vanishes for the symmetric hysteresis loop, was found to assume nonzero values for some range of values of field amplitude $\left(h_{0}\right)$ and temperature $(T)$, dependent on the frequency $(\omega)$. The $h_{0}-T$ plane is then divided by a phase boundary line which separates the dynamically disordered $(Q=0)$ phase from a dynamically ordered $(Q \neq 0)$ phase for any fixed frequency $\omega$. Tomé and de Oliveira (1990) also identified a tricritical point on the phase boundary line which separates the continuous/ discontinuous nature of the transition along the phase boundary. A schematic diagram of the dynamic phase boundary is shown in Fig. 4.

However, as mentioned before, in this mean field approximation the dynamic transition can exist even in the static limit! The reason is that for field amplitudes less than the static coercive field $h_{c}$ (which is nonzero below the order-disorder transition temperature $T_{c}$ ), the response magnetisation varies periodically but asymmetrically even in the zero frequency limit. The system then remains locked to the higher, yet locally attractive, well of the free energy and cannot go to the other (deeper) well, unless driven by any noise or fluctuations which are absent in the mean field system.

This mean field dynamic transition phase boundary in the static limit can be easily estimated. This is because, the $h_{0}(T)$ line in the $h_{0}-T$ plane for $\omega=0$ corresponds to the temperature dependence of the static coercive field $h_{c}(T)$. Since the Landau (mean field) free energy grows as $\left(T_{c}-T\right)^{2}$ for $T<T_{c}$, and the spontaneous magnetisation is $m_{0} \simeq\left(T_{c}-T\right)^{1 / 2}$, the coercive field can be estimated from the balance of $m_{0} h_{c}$ with the free energy barrier height: $m_{0} h_{c} \sim\left(T_{c}-T\right)^{2}$, or $h_{c} \simeq\left(T_{c}-T\right)^{3 / 2}$. The mean field dynamic phase transition boundary (Acharyya and Chakrabarti 1994) indeed converges to such a behaviour in the static limit: $h_{0}(T) \simeq\left(T_{c}-T\right)^{3 / 2}$.

\section{Thermodynamic nature of the transition:}

Lo and Pelcovits (1990) first attempted to study the dynamic transition in the presence of fluctuations as in the kinetic Ising model, using Monte Carlo simulations. While they could 
detect the transition, they could not obtain any precise phase boundary for the transition. Acharyya and Chakrabarti (1994) obtained later the dynamic transition phase boundary. Afterwards, various studies have been made to investigate the thermodynamic nature of this transition.

Extensive Monte Carlo studies in two and three dimensions show that a precise phase boundary (in the $h_{0}-T$ plane) exists for the transition at any fixed nonzero frequency $(\omega)$ of the driving field. For $\left(h_{0}, T\right)$ values below this boundary, one gets asymmetric $m-h$ loops (or asymmetric dynamic hysteresis loops), while for values above this boundary the loops are symmetric. This spontaneous symmetry breaking transition of the (dynamic) hysteresis loop can be accurately described by the behaviour of the dynamic order parameter $Q$, which measures the stable (long time) average value of the magnetisation over a complete period. As mentioned before, this dynamic breaking of symmetry arises due to the competing time scales of the oscillating field and that of the response magnetisation. With decreasing frequency, the phase boundary line in the $h_{0}-T$ plane shrinks towards the origin, and eventually it becomes a line along the $T$-axis $\left(h_{0}=0\right)$, ending at $T=T_{c}$. Recently, Acharyya (1998b) identified this dynamic transition point as the point where the correlation between $h(t)$ and $m(t)$ goes to a minumum.

The Monte Carlo studies also indicate that the transition clearly becomes discontinuous ( $Q$ discontinuously changes) for low temperatures and high field amplitudes at any fixed frequency of the driving field (see insets of Fig. 5). These indications suggest the existence of a tricritical point on the phase boundary which separates the continuous/discontinuous nature of transitions. Very recently, Acharyya (1999) has checked the existence of this tricritical point by studying the distribution $P(Q)$ of the order parameter $Q$ and the temperature variation of its fourth order cumulant $\left(U_{L}=1.0-\frac{<Q^{4}>}{3<Q^{2}>^{2}} ;\right.$ where $\left.<Q^{n}>=\int Q^{n} P(Q) d Q\right)$ across the phase boundary.

Notwithstanding the fact that the response magnetisation $m(t)$ is not necessarily sinusoidal, although it is periodic with the same frequency as that of the external sinusoidally varying field $h(t)$, Acharyya and Chakrabarti (1994, 1995) defined an AC susceptibility 
$\chi=m(t) / h(t) \sim\left(m_{0} / h_{0}\right) \exp (-i \phi)$, assuming a representation $m(t)=m_{0} \exp \left[i \omega\left(t-\tau_{\text {eff }}\right)\right]$ for $h(t) \sim h_{0} \exp (i \omega t)$. Here, $m_{0}$ corresponds to the amplitude of the response magnetisation and $\phi \equiv \omega \tau_{\text {eff }}$ is the effective phase lag (given by the effective delay time $\tau_{\text {eff }}$ of the response $m(t)$ compared to the field $h(t))$. Their Monte Carlo study for two and three dimensional lattices on the temperature variation of this complex susceptibility (moduli of the real part $\chi^{\prime}=\left(m_{0} / h_{0}\right) \cos \left[\omega \tau_{e f f}\right]$ and the imaginary part $\left.\chi^{\prime \prime}=\left(m_{0} / h_{0}\right) \sin \left[\omega \tau_{e f f}\right]\right)$ of an Ising model in a periodically-varying external field shows that the prominent peak in $\chi^{\prime \prime}$ (and dip in $\chi^{\prime}$ ) occurs at $T_{d}$ as one crosses the dynamic phase boundary $\left(Q \neq 0\right.$ for $T<T_{d}\left(h_{0}, \omega\right)$ and $Q$ $=0$ for $T \geq T_{d}$ ). It may be noted that this $\mathrm{AC}$ susceptibility does not (directly) measure any asymmetry of the $m-h$ loops as $Q$ measures: it essentially measures the delay $\tau_{\text {eff }}$ in $m(t)$ compared to $h(t)$. Yet, the AC susceptibility measurements give precisely the same dynamic symmetry breaking transition, where they show peaks or dips. This feature of the AC susceptibility is of course observed only for small values of $h_{0}$ (and large values of $T$ ), where the dynamic transition is continuous.

Recently, the relaxation behaviour of the dynamic order parameter has been studied (Acharyya 1997a). Starting from any arbitrary initial state in the Monte Carlo study, one can study the relaxation behaviour of $Q(p)$, which represent the value of $Q$ for the $p$-th cycle. Acharyya showed that, similar to the critical slowing down behaviour in static critical phenomena, here also the dynamics becomes extremely slow (in $p$ ) as one approaches the dynamic phase boundary $T_{d}\left(h_{0}, \omega\right)$. He also showed (Acharyya 1997b) that the fluctuations $\delta Q$ in $Q$, over its average value, tend to diverge as one approaches the phase boundary (see Fig. 6). Very recently, Sides et al (1998c) applied a finite size scaling method ${ }^{\text {f }}$ to

\footnotetext{
${ }^{3}$ In normal (static) critical phenomena, as one approaches the transition point $T_{c}$, the correlation length $\xi_{s}$ diverges: $\xi_{s} \sim\left|T-T_{c}\right|^{-\nu_{s}}$. In a finite system of linear size $L$, one observes a pseudocritical point at $T_{c}^{e}$ where $\xi_{s}$ becomes equal to $L: T_{c}^{e}-T_{c} \sim L^{-1 / \nu_{s}}$. The finite size scaling method utilises this scaling property of $T_{c}^{e}$ with $L$ and estimates the values of the correlation length and
} 
the Monte Carlo results for the dynamic transition in the two dimensional Ising model and estimated the values of several exponents. In particular, their findings suggest that the correlation length $\xi \sim\left|T-T_{d}\right|^{-\nu}$ for this dynamic transition grows with an exponent value $\nu \simeq 1.1$, slightly higher than the corresponding static transition exponent value $\left(\nu_{s}=1\right)$ in two dimensions.

\section{B. Due to pulsed and stochastic fields}

Since the oscillating field can be decomposed structurally into successive applications of positive and negative field pulses, considerable insights can be gained by investigating the nature of the dynamic response to isolated pulsed fields. For the individual application of pulses, the "positivity" or "negativity" corresponds to fields favouring or competing with the existing order before the application of the pulse respectively. Another interesting study of dynamic response has been in the case of a stochastically varying (in time) field, where the entire extended system is in a (spatially) uniform field which varies randomly in time. The relaxation time of the extended system being much larger than the time unit over which the field changes randomly, a dynamically broken symmetric phase again appears.

\section{(a) Response to a positive pulse field:}

Acharyya et al (1997) studied the response of the kinetic Ising model (1a) to a pulsed field $h(t)=h_{p}$ for $t_{0} \leq t \leq t+\delta t$ and $h(t)=0$ elsewhere. Here $h_{p}$ is in the direction of the existing magnetic order $m_{e}(T)$ for $T<T_{c}$ and $t_{0}$ is much larger than the relaxation time of the unperturbed system at that temperature. The behaviour of the response magnetisation $m(t)$ was studied as a function of temperature $T$, pulse height $h_{p}$ and pulse width $\delta t$, using Monte Carlo simulations and also solving numerically the mean field equation of motion (2), with $h(t)$ as given above. In particular, the response contribution to the magnetisation was characterised by a (maximum) height $m_{p}$ and a width $\Delta t$. For small values of $h_{p}$, the width

other exponents. 
ratio $R \equiv \Delta t / \delta t$ was found to diverge at the critical point $T_{c}$ of the unperturbed system, while the pulse susceptibility $\chi_{p} \equiv m_{p} / h_{p}$ was found to show a finite peak at an effective transition temperature $T_{c}^{e} \neq T_{c}$. It was found that the peak height increases and the peak position $T_{c}^{e}$ approaches $T_{c}$ as the pulse width $\delta t$ increases: $T_{c}^{e}=T_{c}+\mathrm{C}(\delta t)^{-x} ; x \simeq 0.5$ in two dimensions (Acharyya et al 1997). It was argued that this effective transition with a finite peak at $T_{c}^{e}$ occurs due to a finite time effect, similar to the finite size effect in static critical phenomena (Fisher 1964; see footnote 3) : one observes an effective critical behaviour here when the width of the time window $\delta t$ becomes equal to the relaxation time of the order of $\xi_{s}^{z} \sim\left|T_{c}^{e}-T_{c}\right|^{-\nu_{s} z}$, or $T_{c}^{e} \sim T_{c}+\mathrm{C}(\delta t)^{-x}$ with $x=1 / \nu_{s} z$, where $\nu_{s}$ and $z$ denote the standard correlation length and dynamic exponents respectively for the equilibrium transition at $T_{c}$. Indeed, the value of $\nu_{s} z$ is about 2.0 for two-dimensional Ising systems.

Study of the response to such pulsed perturbations in various self-organised critical models can help to predict the critical point before the critical threshold is reached (Acharyya and Chakrabarti 1996). Many of the catastrophic disasters occur dynamically in a self-organised way and the disaster point (threshold stress or time) may often be identified as a critical point. Naturally, the estimate (prior to the disaster) of such self-organised critical points are extremely useful. Since most such dynamically self-organised models respond to the appropriate perturbations, which in turn accumulate to take the system towards the critical point, the perturbations to test the interval from the criticality must necessarily be local in space and time (i.e., pulsed). Measuring the characteristics of the response to local pulsed perturbations, one can extrapolate or predict the imminent disaster point (time) in e.g., some self-organised criticality models of earthquakes (see e.g., Chakrabarti and Benguigui 1997).

\section{(b) Response to a negative pulse field and the magnetisation-reversal transition:}

Misra and Chakrabarti $(1997,1998)$ studied the response of the kinetic Ising model (1a) to a negative pulsed field $h(t)=-h_{p}$ for $t_{0} \leq t \leq t_{0}+\delta t$ and $h(t)=0$ elsewhere. They used Monte Carlo simulations and numerical solutions of the mean field equation of motion (2) 
with the above form of $h(t)$. Due to the application of the negative field pulse $h_{p}$, which opposes the existing order characterised by the equilibrium magnetisation $m_{e}(T)$ for $T<T_{c}$, the down-spin domains start growing and continue until the field is withdrawn. Depending on the average magnetisation $m\left(t_{0}+\delta t\right)$ at the time of the field withdrawal, the domains either grow further (for negative values of $m\left(t_{0}+\delta t\right)$ ) to reach eventually the other equivalent ordered phase with reversed magnetisation $-m_{e}$ or they settle down (for positive values of $\left.m\left(t_{0}+\delta t\right)\right)$ to the original ordered phase with magnetisation $m_{e}$. This transition between the two well-known equilibrium states, driven by pulsed fields competing with the existing order of any of the equivalent states, is essentially dynamic in nature and has interesting properties.

Misra and Chakrabarti studied the phase diagram in the $h_{p}-\delta t$ plane for this dynamic magnetisation-reversal transition (from the phase with equilibrium magnetisation $m_{e}(T)$ before the application of the negative pulse, to the equivalent phase with magnetisation $-m_{e}(T)$ after the application of the field) for different temperatures $T$ below $T_{c}$. The phase boundary here gives the minimal combination of the negative pulse strength $\left(h_{p}\right)$ and width $(\delta t)$ required for the magnetisation-reversal transition. It is seen that the average magnetisation $m\left(t_{0}+\delta t\right)$ at the time of withdrawal of the pulse is the appropriate order-parameter for such a transition and the average relaxation time required for $m(t)$ to settle to the final equilibrium magnetisation $m_{e}(T)$ or $-m_{e}(T)$ diverges as one approaches the phase boundary (from either side, at any fixed temperature below the static critical temperature $T_{c}$ ). This observation strongly indicates the thermodynamic nature of this dynamic transition under a negative pulsed field.

We discussed above such magnetisation-reversal transitions in the presence of fluctuations (e.g., in the Monte Carlo studies). One can also study the mean field version of such a transition, where some analytic estimate can be made for the phase boundary. It may be noted that a major qualitative difference exists between the phase diagrams of the respective cases: while in the presence of fluctuations $h_{p}(T, \delta t) \rightarrow 0$ for the phase boundary for the pulse width $\delta t \rightarrow \infty, h_{p}(t, \delta t)=h_{c}(T)$ for $\delta t \rightarrow \infty$ in the mean field case where the 
static coercive field $h_{c}(T)$ is nonvanishing for $T<T_{c}$. For small $\delta t$, the mean field phase boundary can be estimated approximately by solving the linearised mean field equation (4) with $h(t)=-h_{p}$ for $t_{0} \leq t \leq t_{0}+\delta t$ and $h_{p}=0$ elsewhere. This linearisation is most appropriate for $t \lesssim t_{0}+\delta t$, and the solution can be written as

$$
m(t) \sim\left(m_{e}+\frac{h_{p}}{\epsilon T}\right) \exp \left[-\epsilon\left(t-t_{0}\right)\right]-\frac{h_{p}}{\epsilon T} ; \quad \epsilon=-\frac{1-T}{T} .
$$

One can, in fact, check this solution by direct substitution in (4), with the above form of $h(t)$. The magnetisation-reversal transition occurs if $m\left(t_{0}+\delta t\right) \leq 0$. The phase boundary can therefore be obtained from the solution of $m\left(t_{0}+\delta t\right)=0$. This gives $h_{p} \delta t \simeq m_{e} T$, for the equation of the phase boundary for $T \simeq T_{c}=1$ and small $\delta t$ (or large $h_{p}$ ). In this region, the above phase boundary equation in fact agrees fairly well with even the Monte Carlo phase diagram. When the contributions of fluctuations become important, the above mean field theory fails. If $h_{p} \rightarrow 0$ (as in the large $\delta t$ region of the phase diagram), one can again use the picture of nucleation of a single domain. Equating the growth rate given by the Becker and Döring nucleation rate $I$ (discussed in section III) with the inverse pulse width, one gets $\delta t \simeq \exp \left(1 / h_{p}^{d-1}\right)$, suggesting $h_{p} \ln \delta t=$ constant along the phase boundary in two dimensions. This agrees fairly well with the Monte Carlo estimated phase diagrams in the same dimensions, except at very low temperatures where larger fields induce crossover to multi-domains (Misra and Chakrabarti 1997, 1998, Acharyya and Stauffer 1998).

\section{(c) Dynamic transition due to random (time varying) fields:}

Very recently, an interesting version of this dynamic phase transition has been predicted for a highly anisotropic (Ising-like) magnetic system when the external field on the system varies in time stochastically. Acharyya (1998a) studied the long time response (magnetisation) of a kinetic Ising system represented by the Hamiltonian (1a) when the uniform field over the sample $h(t)$ varies randomly in time with a white distribution bounded between $+h_{0} / 2$ and $-h_{0} / 2$. In a Monte Carlo simulation study in two dimension, Acharyya studied the nature of the response magnetisation (see Fig. 7a,b) and determined the dynamic order parameter $Q\left(=(1 / \tau) \int_{0}^{\tau} m\left(t^{\prime}\right) d t^{\prime} ; \tau>>1\right)$ given by the long-time average (over the active duration of 
the magnetic field) of magnetisation. It was found that $Q$ assumes nonzero values below a phase boundary line in the $h_{0}-T$ plane, and vanishes continuously at the transition boundary (see Fig. 7c). Again, the dynamic symmetry breaking transition occurs due to the competing time scales; the relaxation time of the many-body system being larger than the switching time of the random field. Such a dynamic transition is again a nonequilibrium transition, very similar to that for oscillating fields discussed in Section $\operatorname{IV}(\mathrm{A})$. It may be mentioned that, in a slightly different context, a discrete map version of the mean field equation of motion (2) with similar stochastically varying field $h(t)$ was analysed recently by Hausmann and Ruján (1997).

\section{CONCLUDING REMARKS}

The equilibrium response of cooperative systems to external perturbations is now well understood. The nonequilibrium (yet steady state) responses of such systems to time driven perturbations are extremely important technologically and involve intriguing physics. In cases of magnets under oscillating fields, although some of the phenomena have long been used in technology, little was understood until very recently. The scaling properties of dynamic hysteresis and the spontaneous symmetry breaking dynamic transition are now somewhat established and partly understood. The role of stochastic resonance in such dynamic hysteresis phenomena are, although identified, not fully investigated or understood.

The nature of the dynamic transition due to negative pulses, or that occurring for stochastically varying fields in time, are not yet adequately investigated. Although the existence of these transitions are fairly well established, their detailed nature remains poorly understood so far.

Although it is a bit surprising that the interest in such commonly encountered technologically important problems came very late in the day, the subject has developed momentum in the last few years and a lot of interesting physics has already been developed. We conclude with the hope that this brief introductory review on the exciting developments on 
these rather simple and closely encountered dynamical problems in extended systems will inspire further investigations. No doubt, soon one can expect a maturity of the field, leading to new physics and to better utilisation of its technological potential.

Acknowledgements: We thankfully acknowledge the collaborations with J. K. Bhattacharjee, A. Misra, A. K. Sen, D. Stauffer and R. B. Stinchcombe. We are thankful to C. Dasgupta, D. Dhar, R. Pandit, M. Rao, P. A. Rikvold, D. Stauffer and G C Wang for useful comments and suggestions. We would like to thank C. Denniston, A. Dutta, A. Misra, S. Sil and R. B. Stinchcombe for their comments on the manuscript. We are grateful to U. Nowak and M. Staats for their help in using the figure-scanner. Financial supports from the SFB 341 (Cologne University), and Graduiertenkolleg, Struktur und Dynamik Heterogener Systeme (Duisburg University), are gratefully acknowledged by MA. BKC is grateful to the Royal Society, London, for supporting his visit to the Physics Department, Oxford, where a part of this work was done and the final version of this paper was written. 


\section{REFERENCES}

* E-mail: bikas@cmp.saha.ernet.in

c Present address: Theoretische Physik, Universität-Duisburg, D-47048, Duisburg, Germany.

+ E-mail: muktish@thp.uni-duisburg.de

Acharyya M., 1997a, Phys. Rev. E 562407

Acharyya M., 1997b, Phys. Rev. E 561234

Acharyya M., 1998a, Phys. Rev. E 58174

Acharyya M., 1998b, Phys. Rev. E, 58179

Acharyya M., 1999, Phys. Rev. E, (in press; cond-mat/9809011)

Acharyya M., J. K. Bhattacharjee and B. K. Chakrabarti, 1997, Phys. Rev. E 552392

Acharyya M. and B. K. Chakrabarti, 1994, in Annual Reviews of Computational Physics, Vol. I, Ed. D. Stauffer (World Scientific, Singapore) p. 107

Acharyya M. and B. K. Chakrabarti, 1995, Phys. Rev. B 526550

Acharyya M. and B. K. Chakrabarti, 1996, Phys. Rev. E 53 140; ibid 542174

Acharyya M., B. K. Chakrabarti and R. B. Stinchcombe, 1994, J. Phys. A. 271533

Acharyya M. and D. Stauffer, 1998, Euro. Phys. J. B, 5571

Agarwal G. S. and S. R. Shenoy, 1981, Phys. Rev. A, 232719

Benzi R., P. Sutera and A. Vulpiani, 1981, J. Phys. A: Math. Gen. 14 L453

Binder K., 1979, Ed. Monte Carlo Methods in Statistical Physics (Springer, Berlin)

Bray A. J., 1994, Adv. Phys. 43357 
Bruno P., G. Bayreuther, P. Beauvillain, C. Chappert, G. Luget, D. Renard, J. P. Renard and J. Seiden, 1990, J. Appl. Phys. 685759

Chakrabarti B. K. and L. G. Benguigui, 1997, Statistical Physics of Fracture and Breakdown in Disordered Systems (Oxford University Press, Oxford) pp. 128-149

Dahmen K. A. and J. P. Sethna, 1993, Phys. Rev. Lett. 703222

Dhar D. , P. Shukla and J. P. Sethna, 1997, J. Phys. A 305259

Dhar D. and P. Thomas, 1992, J. Phys. A: Math. Gen. 254967

Fan Z. and Z. Jinxiu, 1995, Phys. Rev. Lett. 752027

Feynman R. P., R. B. Leighton and M. Sands, 1964, The Feynman Lectures on Physics, Vol. II (Addision-Wesley, Reading, M. A.) p. 37-5

Fisher M. E., 1964, J. Math. Phys., 5944

Gammaitoni L., P. Hänggi, P. Jung and F. Marchesoni, 1998, Rev. Mod. Phys. 70223

Goldsztein G. H., F. A. Broner and S. H. Strogatz, 1997, SIAM J. Appl. Maths. 571163

Hausmann J. and P. Ruján, Phys. Rev. Lett. 793339

He Y. L. and G. C. Wang, 1993, Phys. Rev. Lett. 702336

Hohl A., H. J. C. Linden, R. Roy, G. Goldsztein, F. Broner and S. H. Strogatz, 1995, Phys. Rev. Lett. 742220

Jiang Q., H. N. Yang and G. C. Wang, 1995, Phys. Rev. B 5214911

Jiang Q., H. N. Yang and G. C. Wang, 1996a, J. Appl. Phys. 795122

Jiang Q., H. N. Yang and G. C. Wang, 1996b, J. Vac. Sci. Tech. 143180

Jung P., G. Gray, R. Roy and P. Mandel, 1990, Phys. Rev. Lett. 651873

Kittel C., 1966, Introduction to Solid State Physics (John Wiley, New York) p. 495 
Landau L. and E. Lifshitz, 1935, Physik Z. Sowietunion 8153

Lo W. S. and R. A. Pelcovits, 1990, Phys. Rev. A 427471

Luse C. N. and A. Zangwill, 1994, Phys. Rev. E 50224

Mahato M. C. and S. R. Shenoy, 1994, Phys. Rev. E 502503

Mallinson J. C., 1987, in Magnetic Recoding, Eds. C. D. Mee and E. D. Daniels, Vol. 1, (Mcgraw-Hill, New York) pp 337-375

Mirollo R. E. and S. H. Strogatz, 1990, SIAM J. Appl. Math. 50108

Misra A. and B. K. Chakrabarti, 1997, Physica A 246510

Misra A. and B. K. Chakrabarti, 1998, Phys. Rev. E, 584277

Paniconi M. and Y. Oono, 1997, Phys. Rev. E 55176

Rao M., H. R. Krishnamurthy and R. Pandit, 1989, J. Phys. Cond. Matt. 19061

Rao M., H. R. Krishnamurthy and R. Pandit, 1990a, Phys. Rev. B 42856

Rao M., H. R. Krishnamurthy and R. Pandit, 1990b, J. Appl. Phys. 67, 5451

Rao M. and R. Pandit, 1991, Phys. Rev. B43, 3373

Rikvold P.A. and B. M. Gorman, 1994, in Annual Reviews of Computational Physics, Vol. I, Ed. D. Stauffer (World Scientific, Singapore) p. 149

Sethna J. P., K. A. Dahmen, S. Kartha, J. A. Krumhansl, B. W. Roberts and J. D. Shore, 1993, Phys. Rev. Lett. 703347

Sides S. W., 1998, Ph.D. Thesis (Florida State University; unpublished)

Sides S. W. , R. A. Ramos, P. A. Rikvold and M. A. Novotny, 1997, J. Appl. Phys. 81 5597

Sides S. W., P. A. Rikvold, and M. A. Novotny, 1998a, Phys. Rev. E 576512 
Sides S. W., P. A. Rikvold and M. A. Novotny, 1998b, J. Appl. Phys. 836494

Sides S. W., P. A. Rikvold and M. A. Novotny, 1998c, Phys. Rev. Lett. 81834

Sides S. W., P. A. Rikvold and M. A. Novotny, 1998d, cond-mat/9809136

Steinmetz C. P., 1892, Trans. Am. Inst. Electr. Eng. 93

Suen J.-S and J. L. Erskine, 1997, Phys. Rev. Lett. 783567

Suzuki M. and R. Kubo, 1968, J. Phys. Soc. Jpn. 2451

Thomas P. B. and D. Dhar, 1993, J. Phys. A: Math Gen 263973

Tomé T. and M. J. de Oliveira, 1990, Phys. Rev. A 414251

Torre E. D., 1966, IEEE Trans. Magn. Audio Electroacoust. 1486

Zheng G. P. and J. X. Zhang, 1998, J. Phys.: Condens. Matter 101863

Zheng G. P. and J. X. Zhang, 1998, Phys. Rev. E 58 R1187

\section{Figure captions}

Fig. 1. Schematic time variation of the response magnetisation $m(t)$ compared to that of the oscillating field $h(t)$ for different values of frequency $\omega$ and amplitude $h_{0}$ of the oscillating field and temperature $T$ of the system. The results are in fact actual Monte Carlo simulation results for an Ising model on a square lattice with the values for $h_{0}$ and $T$ as indicated in the Figures. The Figures on the right hand side show the corresponding $m-h$ loops. The values for loop area $A$ and the dynamic order parameter $Q$ are also indicated in these figures. As one can see, the first two cases correspond to $Q=0$, while the other two correspond to dynamically broken symmetric phase (with $Q \neq 0$ ). The first figure and the last correspond to $A \simeq 0$, while the middle two correspond to nonvanishing $A$.

Fig. 2. Experimental results for the dynamic hysteresis loop area $A$ and the dynamic order parameter $Q$ (Jiang et al. 1995). (a) The results for the loop area $A$ as a function of 
frequency $f$ is plotted at a fixed ac current of 0.4 Amp. The direction of the magnetic field is parallel to the film plane. The insets show plots of $m-h$ loops for the following particular values of the field amplitudes $h_{0}$ : (i) $h_{0}=48.0$ Oe (top inset) and (ii) $h_{0}=63.0$ Oe (bottom inset). (b) The dynamic order parameter $Q$, i.e, the average magnetisation over a cycle, is plotted against the field amplitude at a fixed frequency $f=4 \mathrm{~Hz}$. The insets show plots of $m-h$ loops for the following particular values of the field amplitudes $h_{0}$ : (i) $h_{0}=48.1$ Oe (right inset) and (ii) $h_{0}=12.0$ Oe (left inset).

Fig. 3. Variation of scaled loop area $\tilde{A}=A h_{0}^{-\alpha} T^{\rho}$ with the scaled frequency $\tilde{\omega}=\omega / h_{0}^{\gamma} T^{\delta}$ for the Monte Carlo data in $d=2$. The inset shows the variation of $A$ with $\omega$ at different $h_{0}$ and $T$. Different symbols correspond to different $T$ and $h_{0}:(\circ) T=1.5, h_{0}=1.25 ;(\square)$ $T=1.5, h_{0}=1.5 ;(\triangle) T=2.0, h_{0}=1.25 ;(\diamond) T=2.0, h_{0}=1.5 ;(\star) T=2.5, h_{0}=1.5 ;$ $\left.(\times) T=2.5, h_{0}=1.75 ; \nLeftarrow\right) T=3.0, h_{0}=2.0 ;$ and $(\dagger) T=3.0, h_{0}=2.5$. The solid curve indicates the proposed scaling function $g(\tilde{\omega}) \sim \tilde{\omega}^{\beta} \exp \left(-\tilde{\omega}^{2} / \sigma\right)$ with $\beta=0.3$.

Fig. 4. Schematic diagram of the dynamic phase boundary in the field amplitude $\left(h_{0}\right)$ and temperature $(T)$ plane at a fixed nonzero frequency. The dotted line is the boundary of the discontinuous transition and the solid line represents the boundary of continuous transition. The small circle represents the tricritical point (TCP). Insets demonstrate the breaking of the symmetry of the dynamic hysteresis $(m-h)$ loop due to dynamic transition.

Fig. 5. Phase diagrams in the $h_{0}-T$ plane for various values of $\omega$ gives the functional form of the transition temperature $T_{d}\left(h_{0}, \omega\right)$ for the dynamic phase transition: Monte Carlo results (a) for system sizes $L=100$ in $d=2$, and (b) for $L=20$ in $d=3$. Below $T_{d}\left(h_{0}, \omega\right)$, $Q$ acquires a nonzero value in $\mathrm{F}$ phase and $Q=0$ in $\mathrm{P}$ phase. Different symbols denote different phase boundary lines corresponding to different frequencies $(\omega)$ : $(\square) \omega=0.418$, $(\triangle) \omega=0.208,(\diamond) \omega=0.104$ in $(\mathbf{a})$; and $(\diamond) \omega=0.418,(\square) \omega=0.202$, (॰) $\omega=0.104$ in (b). The locations of the tricritical points (TCP) are indicated by the circle. The insets show the nature of the transition just above (I: $h_{0}=2.2$ and 4.4 in (a) and (b) respectively) 
and below (II: $h_{0}=1.8$ and 3.6 in (a) and (b) respectively) the tricritical points along the phase boundaries.

Fig. 6 Comparison of the temperature variations of the Monte Carlo results in $d=2$, with $L=100, h_{0}=0.2$ and $\omega=0.063: Q($ solid line $),-d Q / d h_{0}(\bullet)$ and $\delta Q^{2}(\boldsymbol{\Delta})$.

Fig. 7. Dynamic transition due to randomly varing fields in time. (a, b) Typical time variation of magnetisation $m(t)$ compared to that of the stochastically varying field $h(t)$ in a Monte Carlo study in $d=2$, with $L=100, T=1.7: h_{0}=3.0$ for (a) and $h_{0}=1.0$ for (b). (c) The corresponding dynamic transition phase boundary (separating the regions with average magnetisations $Q$ zero from nonzero) in the field width $\left(h_{0}\right)$ - temperature $(T)$ plane. The data points are obtained using both sequential updating $(\diamond)$ and random updating (•) in the Monte Carlo simulation. 
Fig. 1
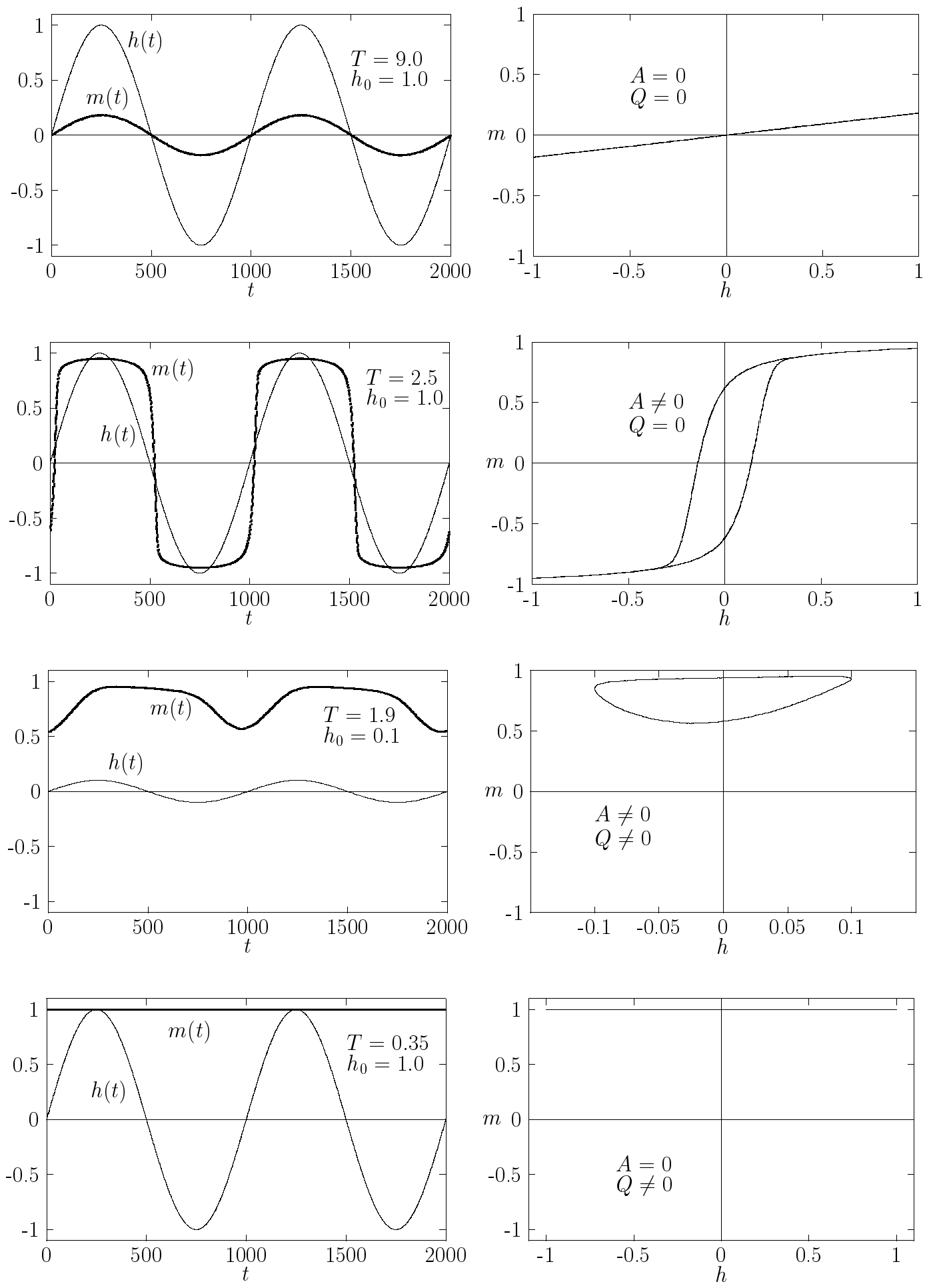


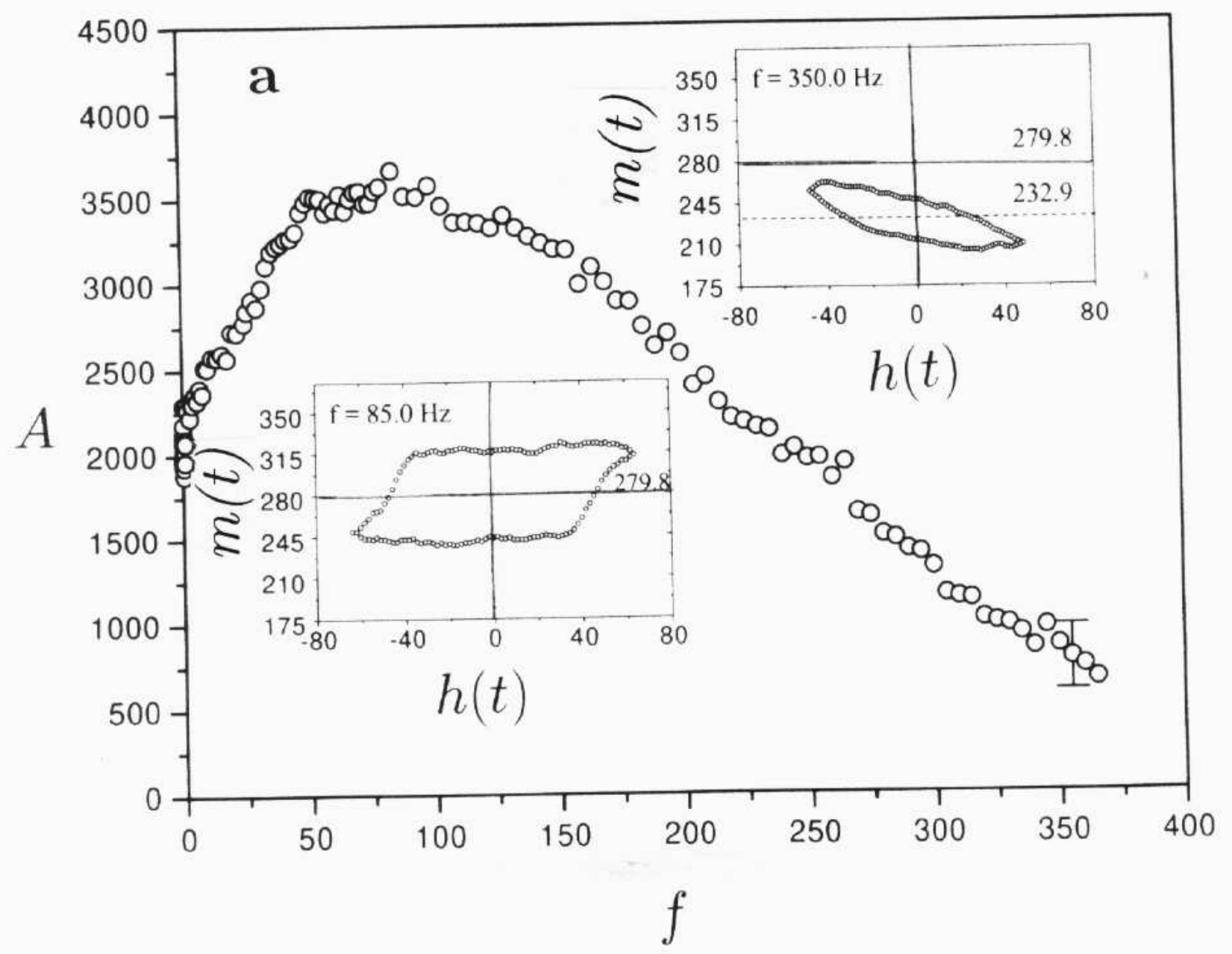

Fig. 2 


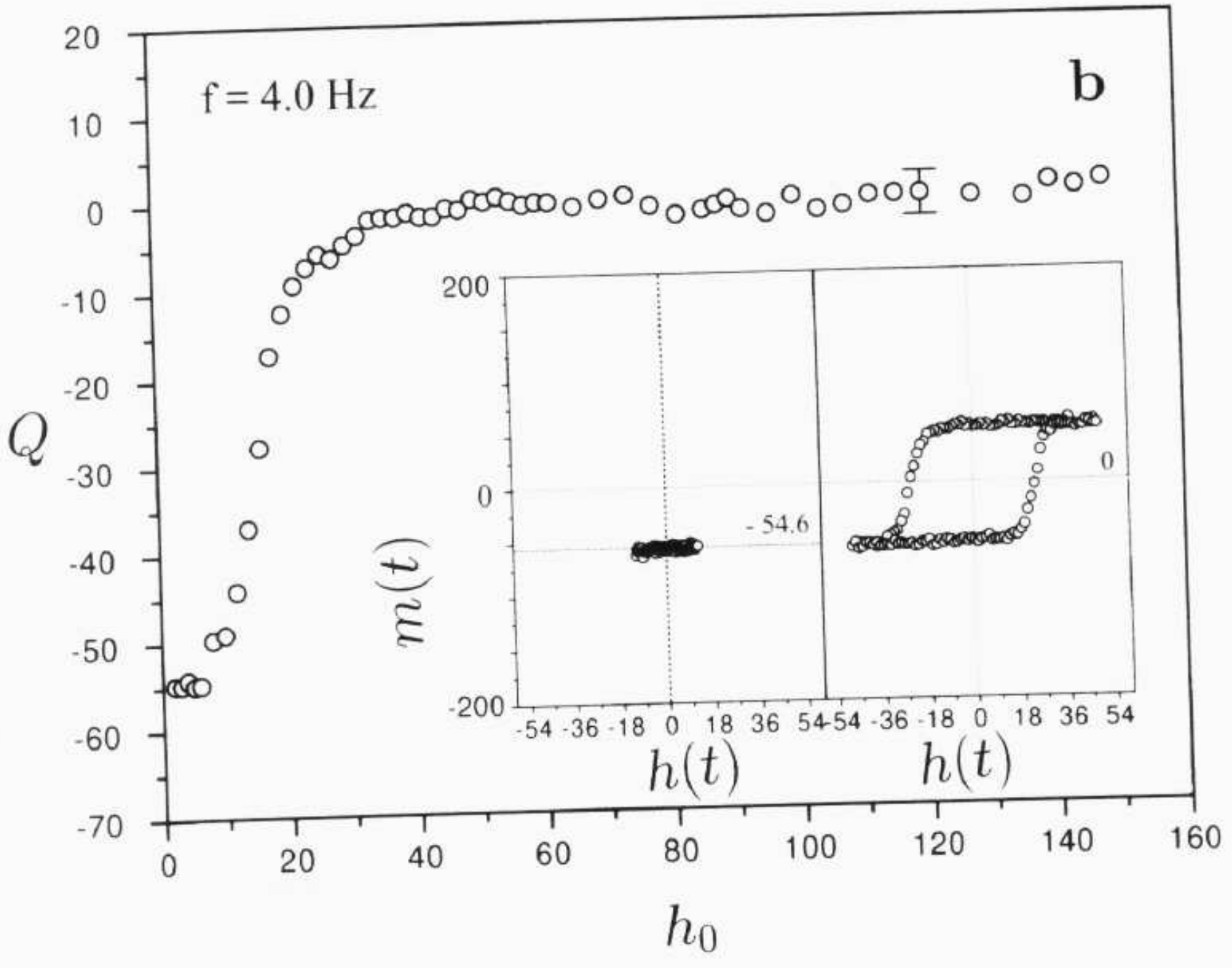

Fig. 2 


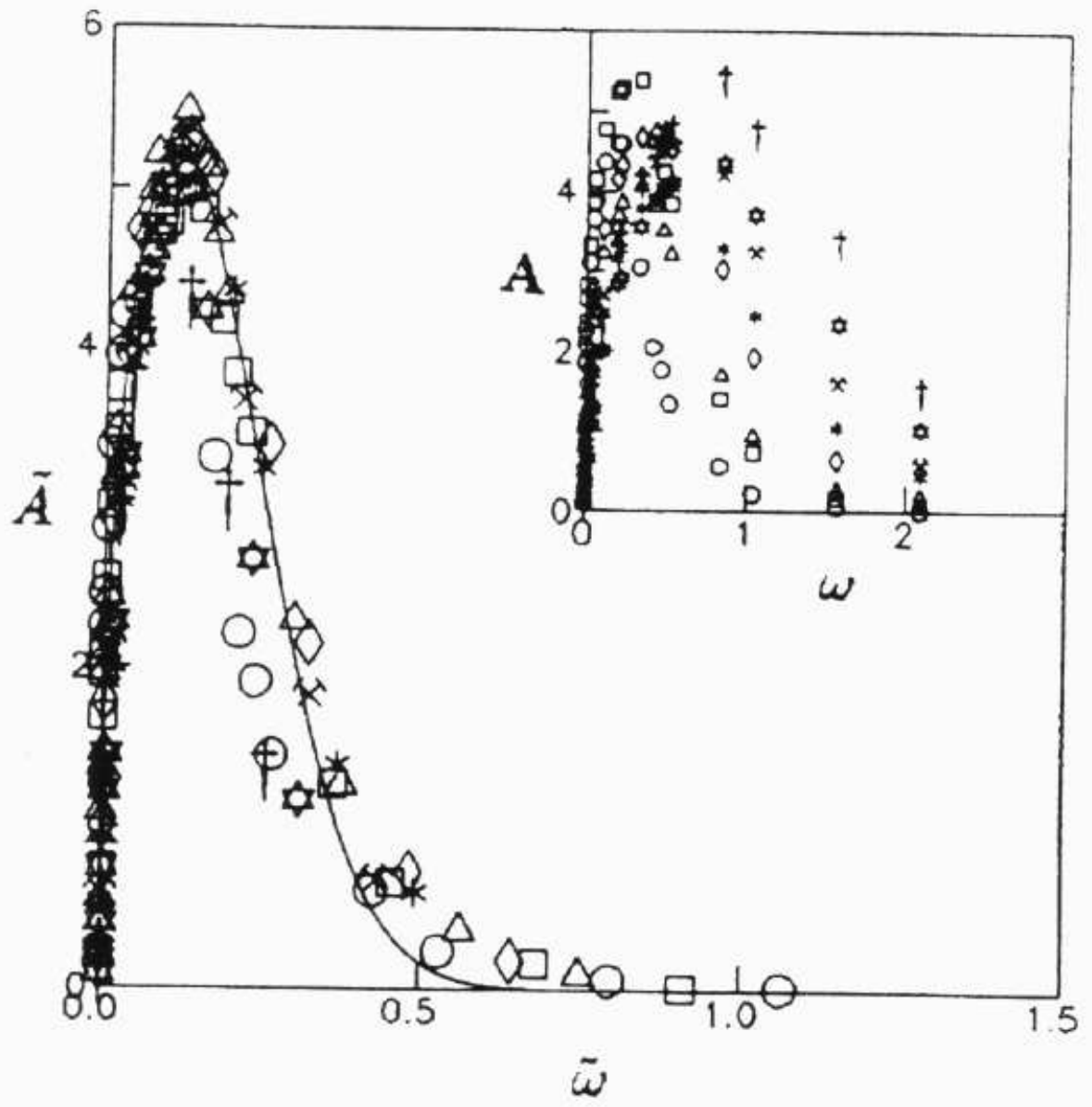

Fig. 3 
Fig- 4

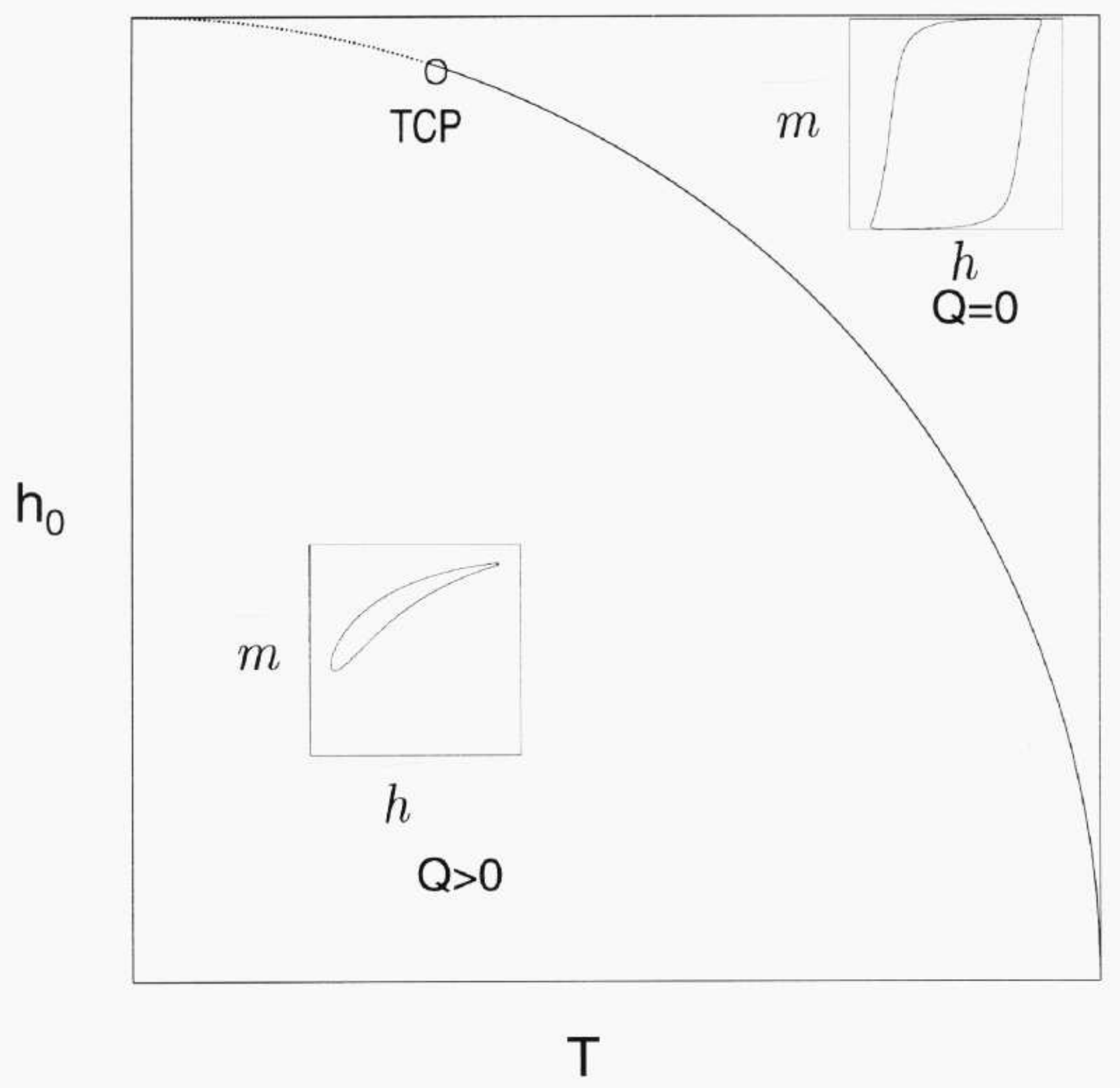



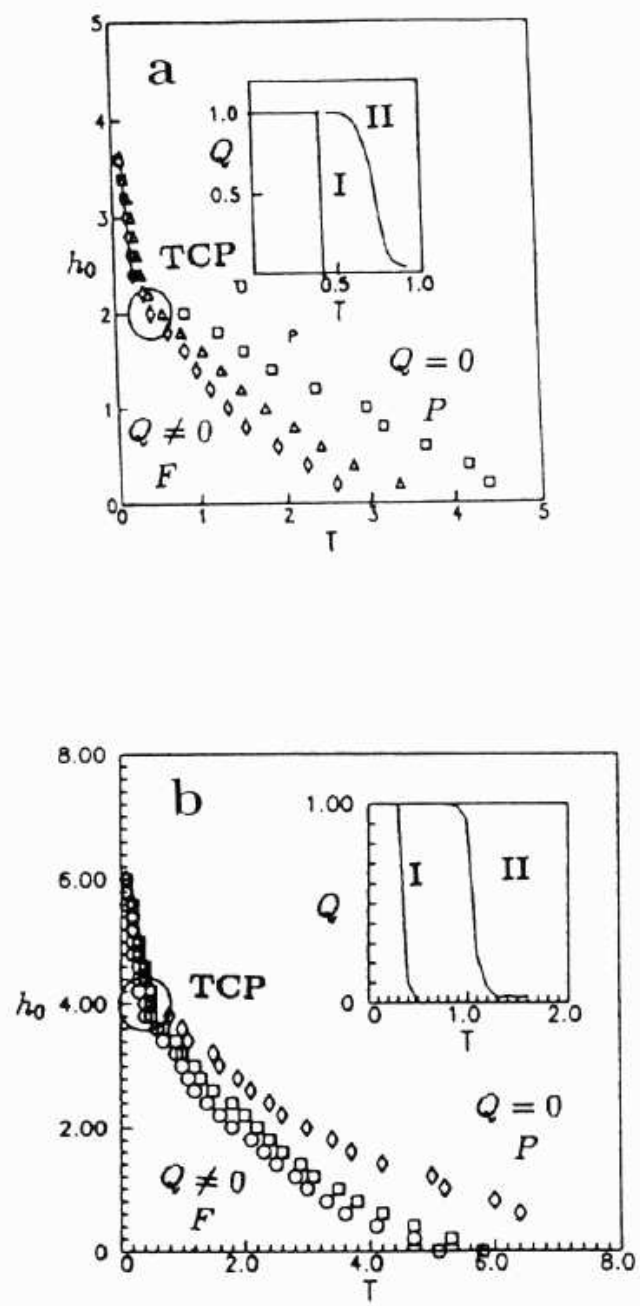

Fig. 5 
Fig- 6

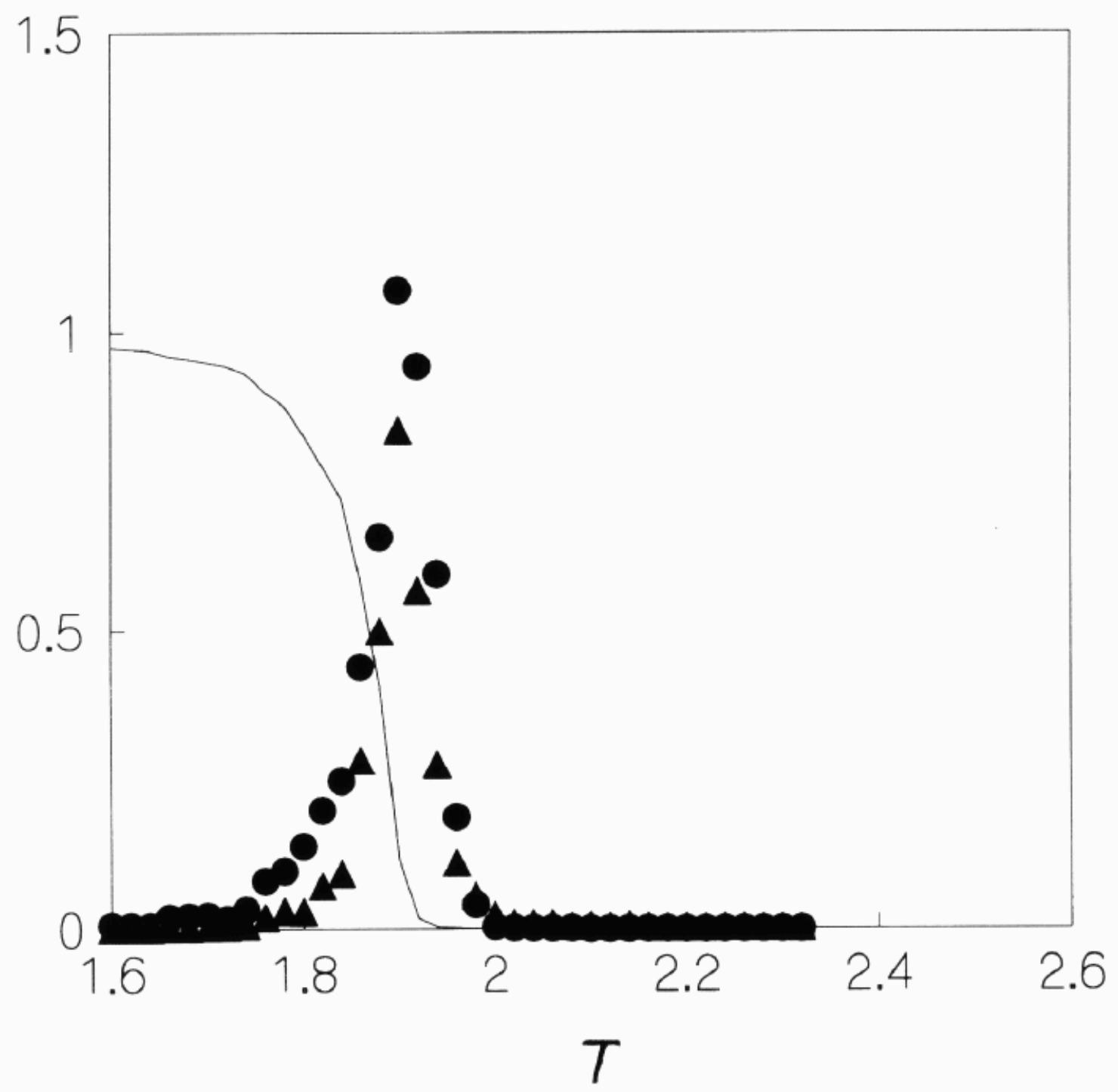



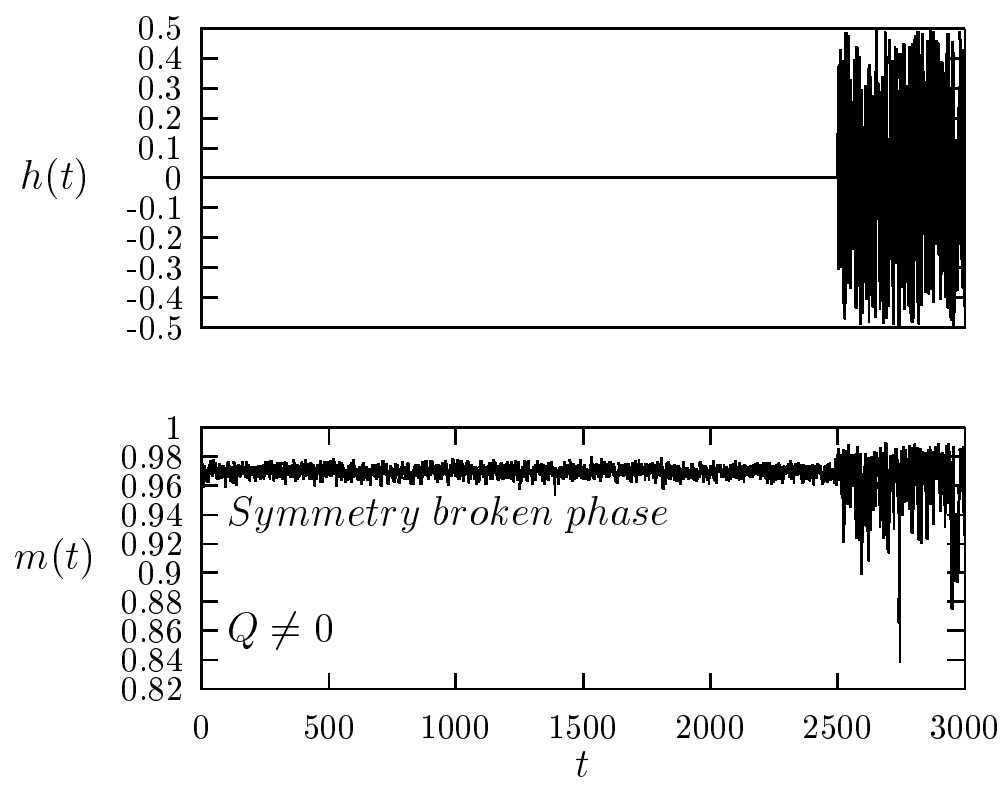

Fig. $7 \mathrm{a}$ 

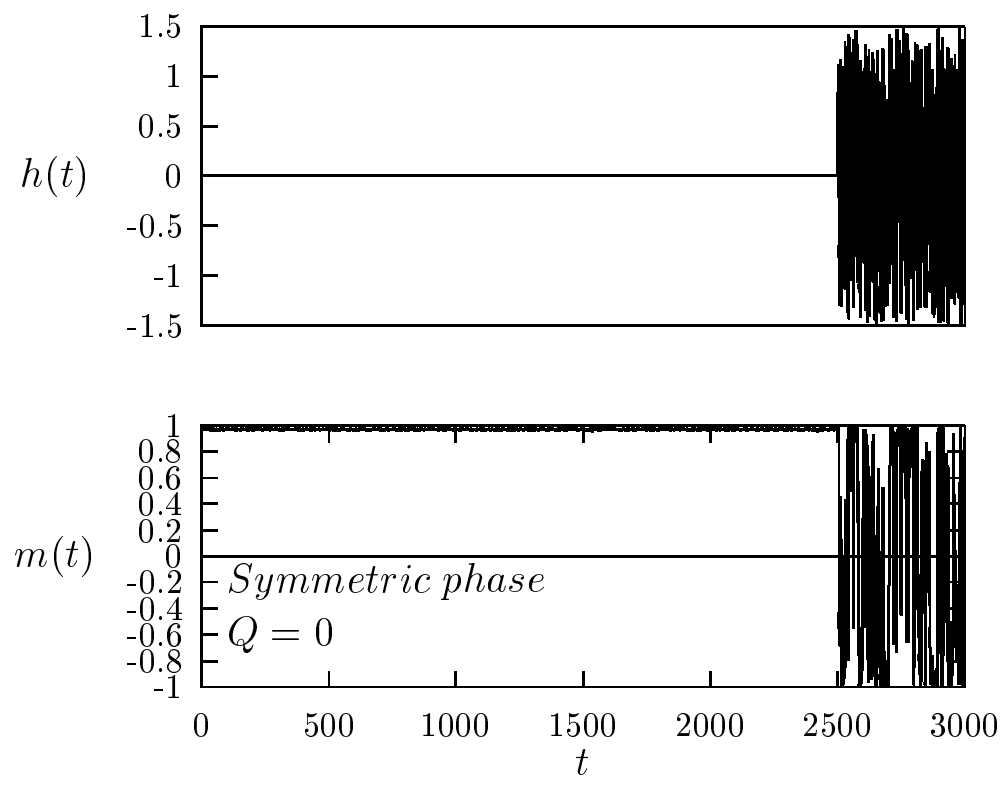

Fig. $7 \mathrm{~b}$ 


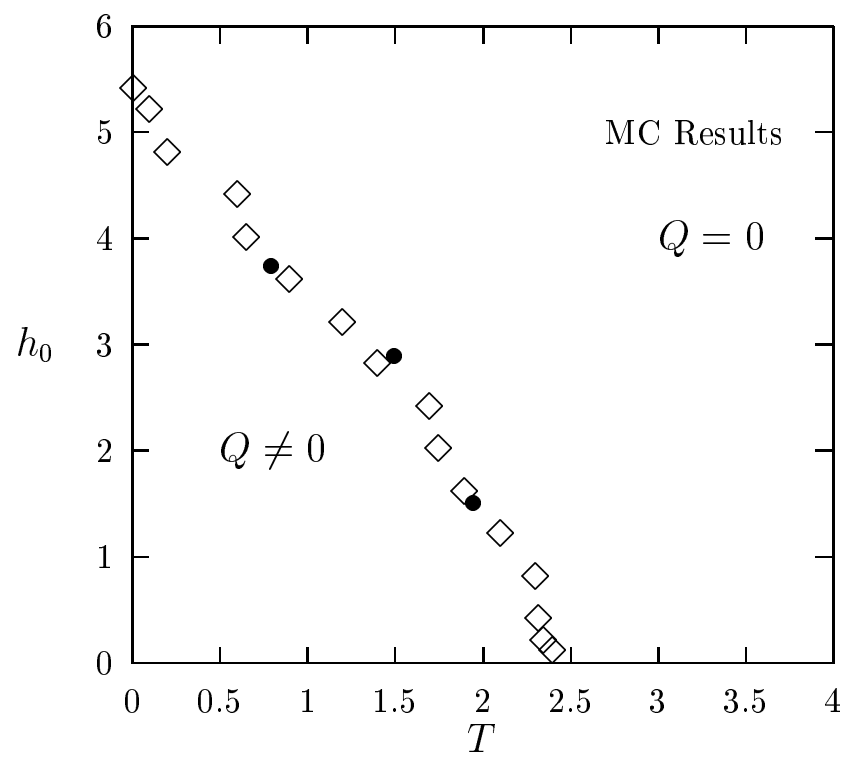

Fig. 7c 